\title{
Design and Research of Urban Haze Weather Monitoring System
}

\author{
Peng Fei Wang ${ }^{a^{*}}$ and Xiaohui Wang ${ }^{b}$ \\ School of leisure management, Xi'an Eurasia University, Xi'an, China

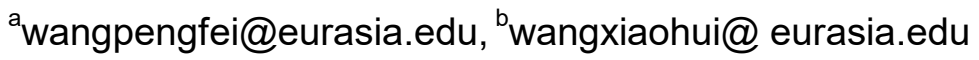

Keywords: Embedded system; environmental monitoring; sensor; system transport.

\begin{abstract}
Nowadays people have higher and higher requirements for the air quality. Using the Tiny6410 development board with the ARM11 processor and the various sensors to realize a haze weather monitoring system. This system can be used for monitoring the temperature, humidity and PM2.5 value of the environment. The collected data can be sent to the PC in real time. Through the test results, it can be seen that the error of the PM2.5 value is small, the temperature and humidity test results are within the actual requirements. So the system can be applied to an unattended area for real-time data acquisition and sending.
\end{abstract}

\section{Introduction}

With the rapid development of the society, the environmental protection has become a major problem that mankind have to deal with in this century [1-2]. With the old indoor testing method, most of the environment data were collected with the large equipment. Although the technology is simple, the precision of the equipment is not high enough. So it is difficult to detect the environmental data in real time [3]. With the continuous development of the technology, the combination of the embedded system and the ARM processor can complete most of the data acquisition and transmission.

In this paper, the urban fog and haze monitoring system which based on ARM processor and Linux operating system was studied. The monitoring system has good application value [4]. It can be widely used in crowded areas such as shopping malls, hospitals and cinemas.

\section{Embedded System Architecture}

Structure of the environmental monitoring system. Based on the ARM11 microprocessor S3C6410 chip connected the sensor and the WiFi interface. Using the embedded Linux system, with the required sensor driver through the cross compiling technology and generate the corresponding Linux kernel. At last, import the kernel into the Tiny6410 board. The surrounding data of the environment is collected by the sensors, and data transmission is accomplished through the Socket network communication.

The development system resource. The system uses the HU-10S as the temperature and humidity sensor, using the GP2Y10 10AU0F as the PM2.5 sensor. The HU-10S sensor transmitted the data through the $\mathrm{I} / \mathrm{O}$ port. With the same reason, the PM2.5 sensor completed the data transmission through the GPIO port.

The embedded Linux system is used as the basic platform. According to the design requirements, the U-boot, the Linux kernel and the file system of the Linux system are properly customized. It facilitated the development of the system. With the wireless USB WiFi driver, the temperature and humidity sensor and the PM2.5 sensor drive, the compressed kernel module is compiled to the Linux kernel with the cross compiled method. So the system is reduced to the minimal. Through the Socket network communication technology and the received data from the reading sensor, programmed the data transfer in the system and realized the information collection and transmission of the monitoring environment.

So, in this paper, using the HU-10S as the temperature and humidity sensor and the GP2Y1010AU0F sensor is used to collect PM2.5 data. 


\section{Debug and Transplant of the Embedded Linux System}

The Linux system is mainly composed of the U-boot, the Linux kernel, the file system and the user program. The embedded U-boot code and the Bootloader program are used to boot the system. The Linux kernel customized the corresponding code according to the actual requirements. The file system mainly contains the system file and the other system commands. Here using the rootfs_qtopia_qt4.img code as the rootfs. The application program includes the necessary drivers and the interaction interfaces that is the desktop of the Qtopia2.2.

Construction of the embedded environment. In the process of the embedded development, in order to run some complex programs that cannot be compiled in the target machine, the executable file must be compiled through the host. After the compilation, the .C file is transplanted into the development board by connecting the host and the development board. So the normal operation of the program was accomplished in the target machine.

The full name of U-Boot is the universal boot loader, which is an open source project with the GPL. The main role of the U-Boot is the system guidance. Most of the U-Boot source code is based on the evolution of the Linux kernel code.

Most of the CPU system structure are accomplished with the assemble language. The first step in the startup of the U-boot is to define the entrance, because the starting point must be unique, so the starting point is defined at $0 \times 0$. The second step is to set the exception vector and adjust the processor's real-time clock, the interrupt registers, and the parameters of the memory control register. The third step is to jump the ROM. Go to the RAM and continue to run.

Embedded Linux kernel transplantation. In actual development, the development board resources are different, so the Busybox code should be reconfigured according to the needs and then use the default configuration file peizhi.config. the Tiny6410 Busybox compiled the configuration file can meet many requirements.

In the Busybox directory, input the command \#cp cppeizhi. config. config and copy the file to the appropriate folder. Then input the "make" command to compilation. The Busybox file is generated after the compilation. It has the same installation and development method as the development board, and busybox is generally not updated.

After entering the Linux system directory, execute the command:

\#mk yaffs2image-128M rootfs_qtopia_qt4 rootfs_qtopia_qt4.img

The rootfs_qtopia_qt4 directory is suppressed as a rootfs_qtopia_qt4.img system image file in yaffs 2 format, which is applicable to the block page organization for the SLC Nand Flash (1 Page $=2 \mathrm{~K}$ Byte, 1 Block=128K). The next step is to download it to the Nand Flash by SD card or USB.

Based on the "ini" index file in the SD card, modified the main definition statements used by the Linux system. Where LCD shares the same zImage document, the super boot used by the system automatically detects the LCD model. The kernel source will add the LED model parameters to the command line. Switch the "S2" of the Tiny6410 to "SDBOOT" and insert the SD card. With the orderly sound, the four green lights on the Tiny6410 development board began to flicker. Finally the buzzer rounded and the LED turns on. So the system had been burnt, as shown in Figure 1.

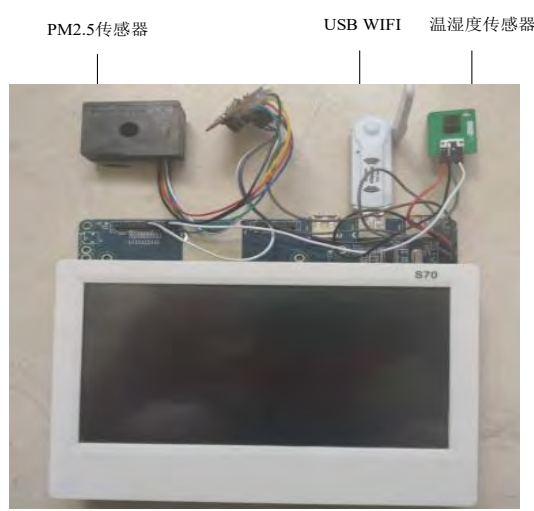

Figure 1. Qtopia2.2.0 system 
Qtopia is a software platform based on Qt development, which is mainly used for embedded Linux systems or mobile phones. Qtopia has a perfect window operating system, games and multimedia, which can shorten the development cycle of the software.

\section{Design the System Software Architecture}

Linux device driver. In the Linux system, most of the sensors are considered as the special external device. The Linux system drives and controls all kinds of sensors through the system. In the embedded Linux system, the control of the sensor is realized by adding the corresponding hardware to the corresponding position. Then the hardware sensor uses the program function code to configure and command the hardware, so that the device can be accessed directly through the embedded Linux. Temperature and humidity sensor program. The program design of the temperature and humidity sensor is divided into two stages. One is running in the kernel, which named as the kernel driver. The other is running in the user program. These two parts complete the data transmission through the file operation function in the user's program. Its specific workflow is shown in Figure 2.

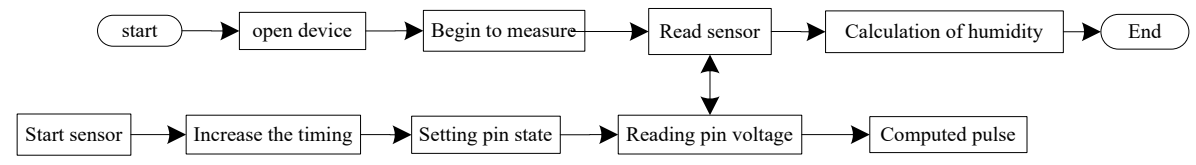

Figure 2. The flowchart of the humidity measurement

Qtopia is a software platform based on Qt development, which is mainly used for embedded Linux systems or mobile phones. Qtopia has a perfect window operating system, games and multimedia, which can shorten the development cycle of the software.

PM2.5 sensor program. The PM2.5 sensor uses the output method of the ADC signal. The Tiny6410 development board used in this article provides a GPIO port and the I/O port. If the detection value is collected, the pulse must be sent to the sensor through the GPIO. By sending a pulse, the ADC can be sampled to the data, thus the concentration of the ambient PM2.5 at the moment is obtained.

Design and implementation of user program. In this paper, the design of the user program is mainly used to realize the function of socket network data transmission. In the embedded Linux, socket network communication is the main way of user access system. By writing the function of network connection and data exchange, the application program can read the sensor.

\section{Measurement Result Analysis}

In order to verify the test results, the school playground, the subway, the hospital, the factory and the outside are tested for five places to be tested. The test results are shown in Figure 3.

In Figure 3, the left is the real-time weather information received by the mobile phone, and the actual test results on the right are on the right. In the outdoor and the playground, the measured data is low; the data of PM2.5 in the subway and hospital crowded places are more than that of the day forecast. The excess of exhaust emissions in the factory can also lead to a steep increase in the number of particles in the area. It can be seen that the test data of the system is stable to PM2.5 and can be used as the source of data for practical testing.

Through the different experiments, the urban haze detection system based on Tiny6410 is very close to the actual environmental data, and can be used as the related requirement of the actual test. 


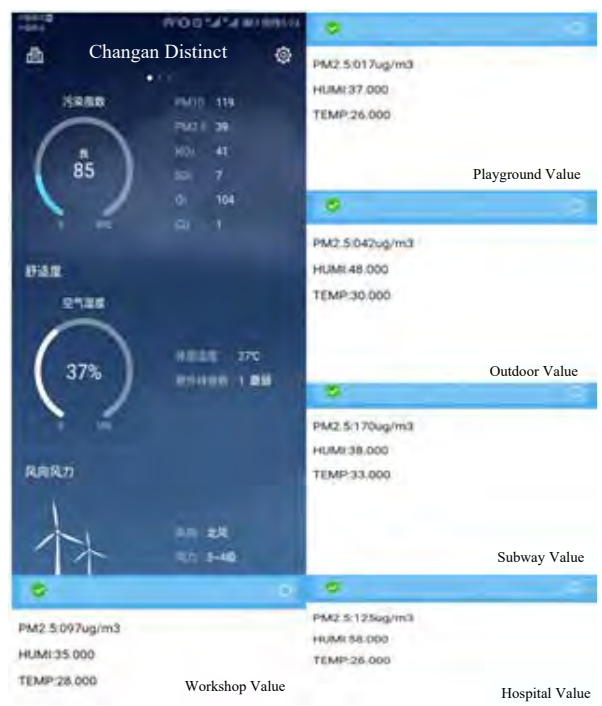

Figure 3. The test results

\section{Conclusions}

The HU-10S and GP2Y1010AU0F sensors are connected to the Tiny6410 development board according to their electrical characteristics and transmission methods. According to Tiny6410 development board and sensor characteristics, add the data collected by the sensor to the Socket network communication based on the Socket network communication, can complete the intended goal of this paper. Through the actual measurement, the Urban Haze detection system designed by this question is running well. The temperature and humidity and the PM2.5 concentration are not much different from the weather forecast, which can better meet the people's higher demand for real time testing of environmental parameters.

\section{Acknowledge}

This paper is sponsored by The Scientific Research Projects of Shaanxi Education Department(18JK1057).

\section{References}

[1] CHANG R, JIANG L H, CHEN W Z, et al. MIPE: a practical memory integrity protection method in a trusted execution environment,Cluster Computing, 20(2017) 1075-1087.

[2] XING R J, JIANG S P, XU P. Long-term temperature monitoring of tunnel in high-cold and high-altitude area using distributed temperature monitoring system ,Measurement,95(2017) 456-464.

[3] SUNG W T, CHEN J H, HSIAO C L. Data fusion for PT100 temperature sensing system heating control model, Measurement, 52(2014) 94-101.

[4] Kim, C G, Kim K J. Implementation of a cost-effective home lighting control system on embedded Linux with OpenWrt,Personal and Ubiquitous Computing, 18(2014) 535-542. 